\title{
Airborne Pathogens Transport in an Aircraft Cabin
}

\author{
M.B. Acikgoz ${ }^{1, a}$, Baris Akay, ${ }^{1, b}$, A.F. Miguel ${ }^{2,3, c}$ and M. Aydin ${ }^{2,4, d}$
}

${ }^{1}$ Dept. Aeronautical Engineering, Istanbul Technical University, 34469 Maslak/lstanbul, Turkey

${ }^{2}$ Geophysics Centre of Évora, Rua Romao Ramalho 59, 7000-671 Évora, Portugal

${ }^{3}$ Dept. Physics, University of Évora, PO Box 94, 7002-554 Évora, Portugal

${ }^{4}$ Institute of Energy, Istanbul Technical University, 34469 Maslak/Istanbul, Turkey

a acikgozbe@itu.edu.tr, ${ }^{\text {b }}$ baris.akay@gmail.com, ${ }^{\mathrm{c}}$ afm@uevora.pt; ${ }^{\mathrm{d}}$ aydinmurat@itu.edu.tr

Key words: Airborne pathogens, dispersion, aircraft cabin, sneezing, coughing, 3D simulation

\begin{abstract}
Airborne pathogens can spread within an aircraft cabin from sneezing, coughing or breathing of a sick passenger. This paper reports a 3D numerical study on the transport of airborne pathogens inside Boeing 767 cabin. After cough or sneeze of an infected passenger, the entire unsteady pathogen dispersion process is simulated. Effects under study include the direction of coughing on the pathogens propagation and the spreading times from infected to hosts passengers.
\end{abstract}

\section{Introduction}

Aircraft travel for both business and leisure purposes has grown strongly worldwide. In 2005, only in USA, airlines carried nearly 600 million total passengers and 500 trillion passenger miles [1]. Flight personnel and passengers travel in an enclosed ventilated aircraft cabin, which exposes travellers to hypobaric hypoxia, dry humidity, and close proximity to fellow passengers during several hours. Most commercial aircraft in service recirculate $50 \%$ of the air delivered to the passenger cabin $[2,3]$. The air usually passes through high efficiency particulate air filters (HEPA) which have a particle-removing efficiency of $99.97 \%$ at 0.3 microns [4].

Airborne diseases are spread when droplets of pathogens are expelled into the air due to coughing, sneezing or even by talking (e.g., coughing or sneezing produce a large number of particles with size up to 100 micron). Large droplet and airborne mechanisms probably represent the greatest risk for passengers within the aircraft [5]. Large droplet transmission takes place when droplets (bigger than 5 micron) contaminated with microorganisms travel short distances (less than one meter) and deposit on a susceptible host's conjunctiva or mucosa (e.g., nasal mucosa). Airborne transmission occurs when small droplets in a warm, dry atmosphere tend to evaporate rapidly and become droplet nuclei (less than 5 micron). These droplet nuclei are the residue of solid material (infectious agent) left after drying up of a droplet. They can remain suspended in air long time and may travel long distances. In addition to proximity, successful spread of contagion to other hosts is dependent of factors such as the duration of exposure; environmental conditions, pathogenicity of the microorganism, host-immune status, etc.. Therefore, spread of contagion of several diseases (e.g., viral diseases such as measles and influenza, bacterial infections such as tuberculosis and others) to other passengers constitutes a significant and realistic threat.

As incubation periods between exposure and contraction of airborne disease vary up to several days, it's difficult to be sure if travellers contracted the disease during the flight. Models for examining infection in confined spaces are generally based on the work of Wells [6] and Riley et al. [7]. In 1988, Brundage et al. [8] showed that febrile illness rates were about 50\% higher in sealedwindow than in operable window army barracks. Wagner et al. [9] estimated that one passenger infected with H1N1 and travelling in economy class of a commercial aircraft may cause two to five infections during a 5 hour flight and a 5-10 during an 11 hour flight. There are also other studies that analyze experimentally and numerically the airborne pollutant transport within aircrafts (see for example, Liu et al. [10], Zhang et al. [11], Mazumdar and Chen [12]). Recently, the effects of a moving human body on flow and contaminant transport inside an aircraft cabin were investigated by Poussou et al. [13]. A recent study on coughed airflows of healthy males asserts that the spread 
velocity of coughing was in the range of $6-22 \mathrm{~m} / \mathrm{s}$ [14]. The study of Zhao et al. indicates that the exhaling process have little influence on the room airflow pattern [15]. Therefore, in the present study, the exhaling of manikins has not taken into account. To shed some light on the transmission of airborne pathogens within a fully occupied aircraft cabin a transient 3D numerical study has been conducted in this paper. A special attention is paid on the direction of expelled pathogens, location of host passengers and spreading times.

\section{Problem Definition and 3D Numerical Study}

Airborne pathogens flows in a tree-dimensional aircraft cabin have been simulated. The various elements of this study are outlined in the following sections.

Aircraft Cabin Model. Airflow and pathogens suspension flow were simulated in a three-row, twin-aisle aircraft cabin with 21 seats fully occupied. The dimensions of the aircraft cabin, seats and passengers (manikins) are given in Fig.1. It consists of tree row of economy-class, twin-aisle cabin similar to a Boeing 767 aircraft. There are two inlets at the ceiling of the aircraft cabin that deliver air to both sides of the passenger cabin. The air is extracted from two outlets located on the side walls at floor level. The seats are fully occupied by passengers and the infected passenger is seated at the middle seat of second row. The width of a seat is $0.53 \mathrm{~m}$. The distance between seats is $0.6 \mathrm{~m}$. The space of the aisle is $0.85 \mathrm{~m}$ and the row's is $1.05 \mathrm{~m}$. The height of a seated manikin is $1.3 \mathrm{~m}$. The total surface area of each manikin is $1.74 \mathrm{~m}^{2}$.
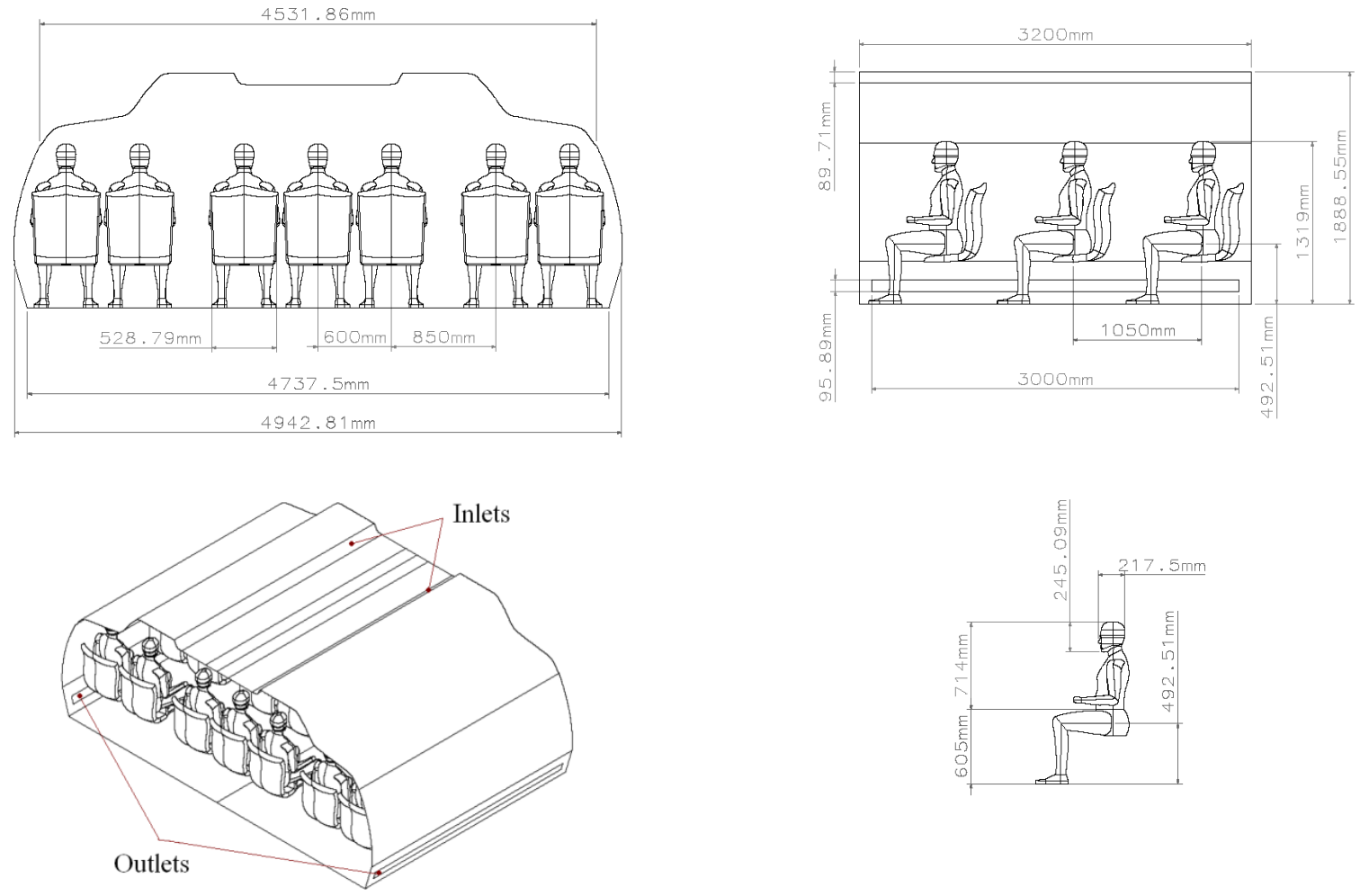

Figure 1 - Aircraft cabin model for 3D simulations.

Governing Equations: Fluid Dynamics of Suspending Fluid. The Newtonian incompressible fluid flow assumed in the cabin model. Thus, the essence of our phenomenological description is the three-dimensional set of Reynolds-averaged Navier-Stokes equations with the turbulent stresses, combined with the energy equation.

Motion of Suspended Airborne Pathogens. Once the airflow field is obtained, the motion of the airborne pathogens in the fluid may be pursued with the Lagrangian type equation of motion [16]. 


$$
\frac{d u_{p}}{d t}=\frac{1}{\rho_{p}}\left[\frac{18 \mu}{d_{p}^{2}} c_{g}\left(u-u_{p}\right)+\frac{1}{2} \rho \frac{d}{d t}\left(u-u_{p}\right)+\xi\left(\frac{216 \rho v \sigma_{B} T}{\pi d_{p}^{5} c_{c} \Delta t}\right)^{1 / 2}+f_{g}\right]
$$

with $c_{g}=\frac{c_{d} \rho d_{p}}{24 \mu}\left|u_{p}-u\right|$ for micrometer and $c_{g}=\frac{1}{c_{c}}$ for submicrometer particles, $u_{p}$ is particle velocity, $\rho$ is the fluid density, $\mu$ is the fluid viscosity, is $u$ the fluid velocity, $c_{d}$ is drag coefficient, $\mathrm{c}_{\mathrm{c}}$ is the Cunningham slip correction factor [4], $\xi$ is the zero-mean unit-variance-independent Gaussian random number, $\sigma_{\mathrm{B}}$ is the Boltzmann constant, $\rho_{\mathrm{p}}$ is the particle density, and $\mathrm{T}$ is the absolute fluid temperature. Here the first right-hand term represents the drag force, the second righthand term is the lift force due to shear, the third right-hand term denotes a force arising from Brownian collisions and the fourth right-hand term represents the effect of force of gravity on the particle. Airborne pathogens trajectories are obtained by integrating this motion of equation [17].

Initial and Boundary Conditions. Initial temperature of the air inside cabin is $292.65 \mathrm{~K}$. Temperatures of cabin ceiling, sidewalls and floor are $299.15 \mathrm{~K}, 297.15 \mathrm{~K}$ and $298.15 \mathrm{~K}$, respectively. The seats are assumed adiabatic and both the temperatures of the passengers and airborne pathogens are $304.15 \mathrm{~K}$. At $\mathrm{t}=0 \mathrm{~s}$, the air enters into the cabin through two inlets located at the ceiling. From inlets, air is introduced at a constant mass flow rate of $0.13 \mathrm{~kg} / \mathrm{s}$ (which equals to $10^{-2} \mathrm{~m}^{3} / \mathrm{s}$ per passenger [18]) in the normal direction to the boundary and at a temperature of 292.65 $\mathrm{K}$. The air is expelled to the outside through the outlets. Non-slip boundary conditions are considered along solid obstacles-fluid interface.

The infected passenger coughs for $0.5 \mathrm{~s}$ with $22 \mathrm{~m} / \mathrm{s}$ coughing velocity and expels $150 \mathrm{mg}$ waterliquid droplets of various sizes (i.e., 1, 10 and $100 \mu \mathrm{m}$ ). The transient numerical simulations were performed to simulate first $60 \mathrm{~s}$. To guarantee the convergence and to obtain more precise solution a time-step of $0.05 \mathrm{~s}$ and 20 iterations for each time-step is adopted for the transient simulations.

Grid Generation and Validation of the CFD Model. In this study, Icem-CFD software was used to build the grid over the complex cabin and manikins (passengers) geometry. A grid independence analysis was performed and various grid sizes were investigated. For the geometry under study, the accuracy was determined by the results of the transient average temperature inside the cabin. The grids for this geometry are composed by coarse, medium and fine computational domains containing 520000, 3.3 million and 7 million tetrahedral elements, respectively. Having obtained satisfactory results for the grid dependency some aspects concerning the dispersion of airborne pathogens were investigated within the aircraft cabin. The computational grid of the cabin contained 7 million tetrahedral elements was found to be appropriate. This grid on the manikin surfaces contains 21000 trilateral elements. The grid was refined in proximity of manikin surfaces, air inlets and outlets regions. (Fig.2)
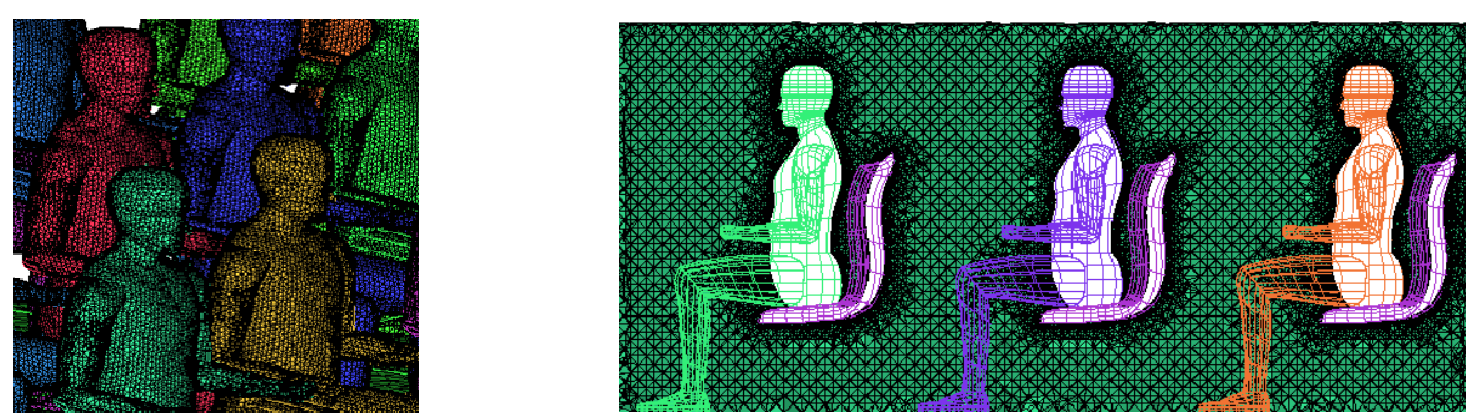

Figure 2 - The grid on the surface of the manikins and in the flow domain (plane view).

Numerical Method. In this study, the computational numerical simulations were performed by a commercial code, Fluent which is based on a finite volume method. Many authors validated the RNG $\kappa-\varepsilon$ turbulent model in various enclosed environments $[11,18,19]$. These results show a good 
agreement with experimental data available in literature. The Reynolds averaged Navier-Stokes equations based on the renormalization-group (RNG) $\kappa-\varepsilon$ turbulent model was adopted to predict the distributions of airflow and temperature. To model the airborne pathogens transport a Lagrangian method was employed. The second-order upwind scheme was used for the momentum equation. The discretization of pressure was based on PRESTO scheme. The SIMPLE algorithm was employed to couple the pressure and momentum equations. The criteria for convergence that are used in the simulations was defined in terms of the residuals which provide a measure of the degree to which each of the conservation equations are satisfied throughout the flow field. Residuals were computed by summing the imbalance in each equation for all cells in the domain. In all of our simulations, convergence is considered to be achieved only when the normalized residuals fall below $10^{-5}$ for mass conservation, $10^{-4}$ for momentum equation and $10^{-7}$ for energy conservation. The results were obtained by a high performance computer with eighteen 64-bit XEON processors of $3 \mathrm{GHz}$ each and $36 \mathrm{~GB}$ of total memory. Each case cost about 3 days to complete $60 \mathrm{~s}$ simulation.

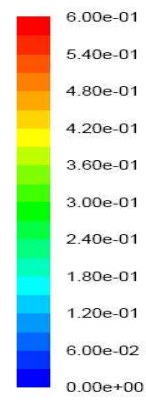

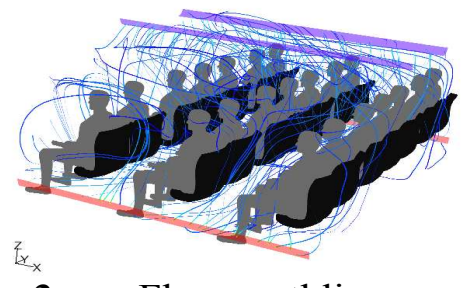

Figure 3a - Flow pathlines velocities $(\mathrm{m} / \mathrm{s})$ within the aircraft cabin with fully occupied seats (isometric view)

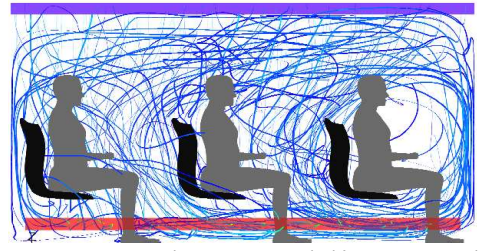

Figure 3b - Flow pathlines velocities $(\mathrm{m} / \mathrm{s})$ within the aircraft cabin with fully occupied seats (side view)

\section{Results and Discussion}

Fig. 3 shows the flow pathlines coloured by air velocity magnitude for the aircraft cabin ventilation. This figure shows a strong mixing effect in the cabin. Therefore, an infected passenger can easily spread the airborne pathogen through the entire cabin.
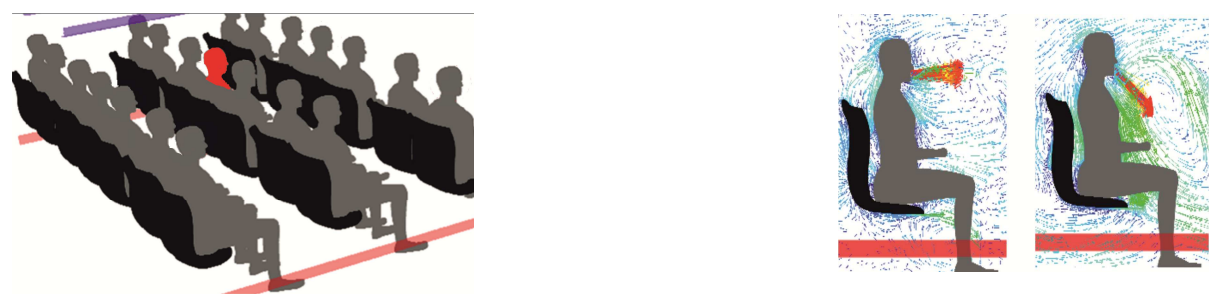

Figure 4 - One infected passenger inside the aircraft cabin and side views of the infected passenger coughing in two different directions: horizontal and oblique (i.e., $-45^{\circ}$ to horizontal direction).

Consider that an infected passenger is seated at the middle seat of second row and may expel airborne pathogens in two different directions (Fig. 4). Figs. 5 and 6 show the propagation of airborne pathogens in time. These plots show that for both coughing directions pathogens of a size of $10 \mu \mathrm{m}$ or less are influenced by the cabin ventilation due to the fact that the effect of gravity negligible. The expel velocity induced by coughing provokes airborne pathogens to travel distances larger than 1.5 meter. These distances depend on coughing direction and particle size (large size particles $(100 \mu \mathrm{m})$ spread in the same direction of coughing due to their inertia). The spreading of particles is facilitated if the pathogens are expelled horizontally (the oblique coughing facilitates the deposition onto the seats and floor). This is especially significant for small particles (droplet nuclei mode). Besides, after $10 \mathrm{~s}$ the concentration of pathogens in the air for horizontal cough is higher than the one for oblique cough. 

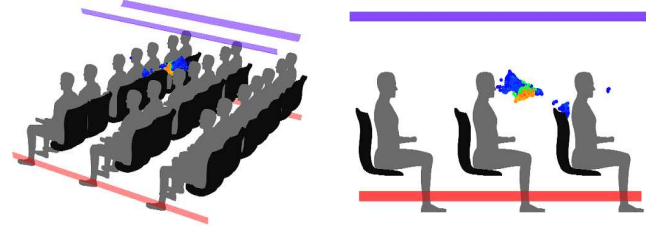

Figure 5a - Particle traces coloured by airborne pathogen diameter $(\mathrm{m})$ for a horizontal cough at time $0.1 \mathrm{~s}$ (isometric and side views)
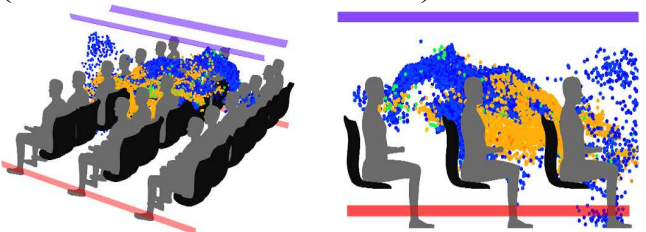

Figure 5b - Particle traces coloured by airborne pathogen diameter $(\mathrm{m})$ for a horizontal cough at time $0.5 \mathrm{~s}$
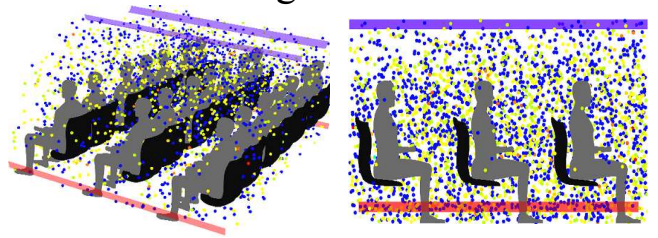

Figure 5c - Particle traces coloured by airborne pathogen diameter $(\mathrm{m})$ for a horizontal cough at time $20 \mathrm{~s}$
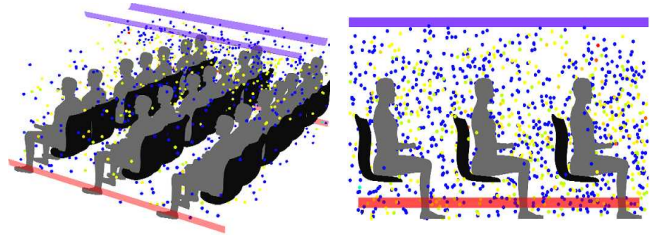

Figure 5d - Particle traces coloured by airborne pathogen diameter $(\mathrm{m})$ for a horizontal cough at time $60 \mathrm{~s}$
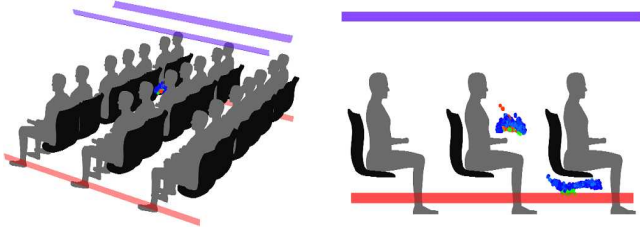

Figure 6a - Particle traces coloured by airborne pathogen diameter $(\mathrm{m})$ for an oblique cough at time $0.1 \mathrm{~s}$ (isometric and side views)
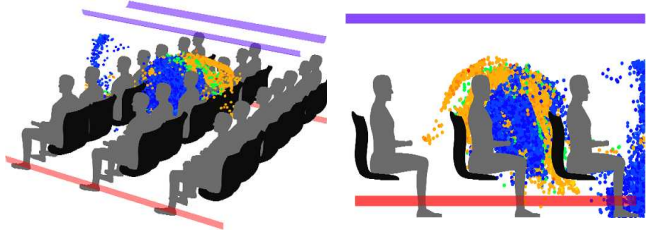

Figure 6b - Particle traces coloured by airborne pathogen diameter $(\mathrm{m})$ for an oblique cough at time $0.5 \mathrm{~s}$
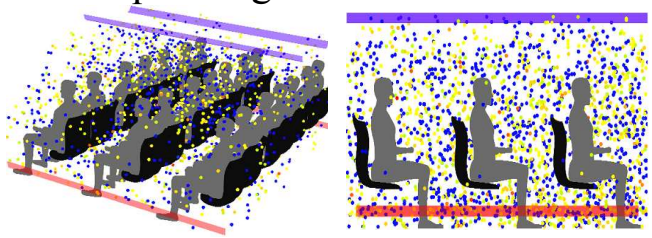

Figure 6c - Particle traces coloured by airborne pathogen diameter $(\mathrm{m})$ for an oblique cough at time $20 \mathrm{~s}$
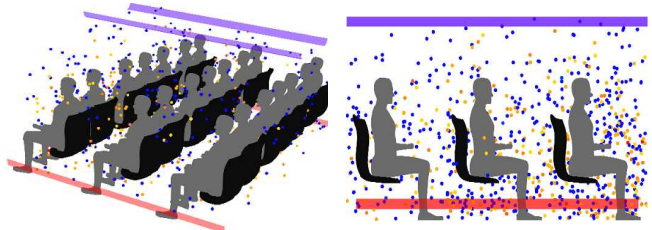

Figure 6d - Particle traces coloured by airborne pathogen diameter $(\mathrm{m})$ for an oblique cough at time $60 \mathrm{~s}$

Table 1 - Exposure of host passengers, $\gamma$, to airborne pathogens.

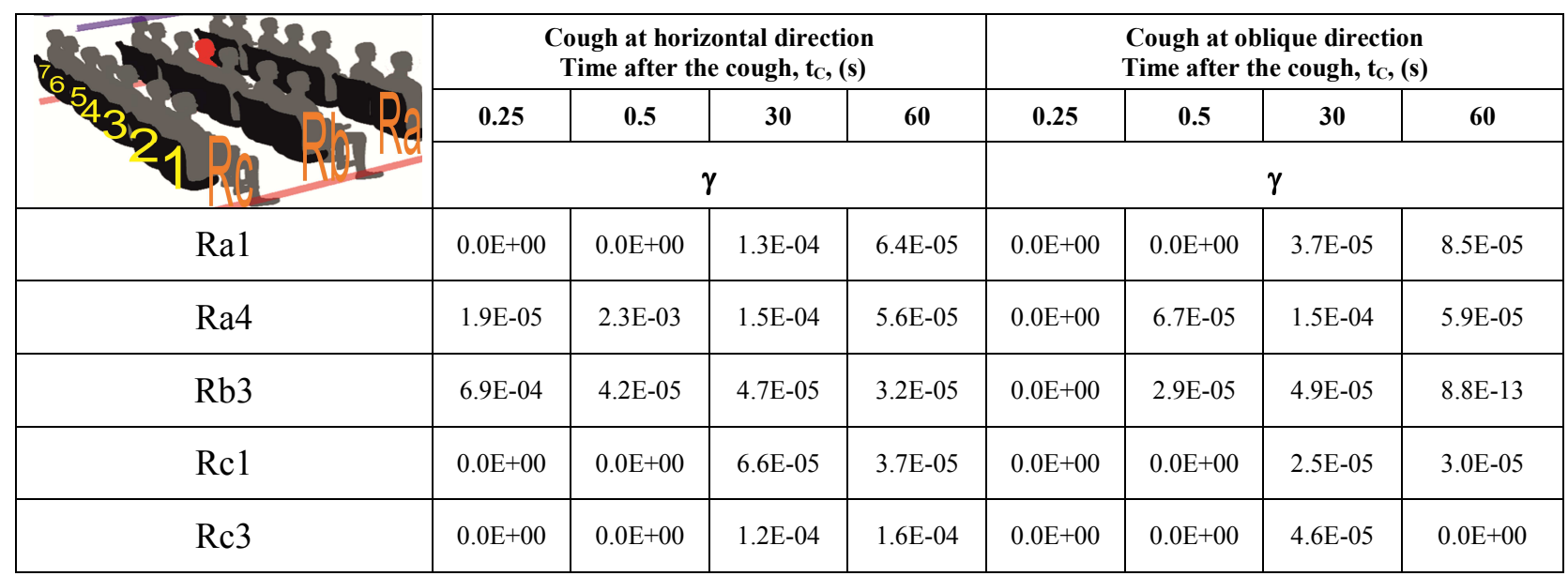

The exposure of the passengers to airborne pathogens, seated at different distances from the infected passenger is tabulated in Table 1 . Their exposures, $\gamma$, were defined by the ratio of mass of pathogens that reach the breathing zone of several host passenger (defined as the ratio of pathogens 
within a volume corresponding to a distance of $15 \mathrm{~cm}$ around the nose) and the mass of pathogens released by the infected passenger. Table 1 shows that, in general, $\gamma$ is smaller for host passengers in case of oblique cough $\left(-45^{\circ}\right.$ in the horizontal direction). Besides, regardless of the passenger distance, $\gamma$ is zero for $t_{C}$ less than $0.5 \mathrm{~s}$. In case of horizontal cough the host passengers at same row seated next/before the infected passenger are more exposed to airborne pathogens. Passenger seated right in front or right behind also has fairly high $\gamma$. In contrast, host passenger seated right in front of the infected passenger has the highest $\gamma$ in case of oblique cough. This data also suggest that regardless of the passenger distance, host passengers seated in the row behind present exposures similar to the one presented by the passenger seated further in the front row.

\section{Conclusion}

This study investigated airflow and airborne pathogens transport in an occupied aircraft cabin by transient 3D numerical simulations. Among other results, the exposure of host passenger to airborne pathogens strongly dependent on seat position to infected passenger and direction of cough.

\section{References}

[1] Bureau of Transportation Statistics: Pocket Guide to Transportation, Table 11 (2005)

[2] M. Withers and G. Christopher: Mil. Med. Vol. 165 (2000), p. 1.

[3] M. Hocking: Chemosphere Vol. 41 (2000), p. 603.

[4] A. Bejan, I. Dincer, S. Lorente, A.F. Miguel and A.H. Reis: Porous and Complex flow Structures in Modern Technologies (Springer, New York, 2004)

[5] J. Fiegel, R. Clarke and D.A. Edwards: Drug Discovery Today Vol. 11 (2006), p. 51.

[6] W. F. Wells: Airborne Contagion and Air Hygiene (Harvard Univ. Press, Cambridge 1955).

[7] E.C. Riley, G. Murphy and R. L. Riley: Am. J. Epidemiol. Vol. 107 (1978), p. 421.

[8] J.F. Brundage, R.M. Scott, W.M. Lednar, et al.: JAMA. Vol. 8 (1988), p. 2108.

[9] B.G. Wagner, B.J. Coburn and S. Blower. BMC Medicine Vol. 7 (2009)

[10] C.H. Lin, R.H. Horstman, M.F. Ahlers et al.: ASHRAE Transactions Vol. 111 (2005), p. 764.

[11] T. Zhang, Q. Chen, and C.H. Lin: HVAC\&R Research Vol. 13 (2007), p.683 and p. 871.

[12] S. Mazumdar and Q. Chen: Indoor Air Vol. 19 (2009), p.3.

[13] S.B. Poussou, S. Mazumdar, M.W. Plesniak, et al.: Atmos. Environ. (2010) in press

[14] S. Zhu, S. Kato and J. H. Yang: Build. Environ. Vol. 41 (2006), p. 1691.

[15] B. Zhao, Z. Zhang and X. Li: Build. Environ. Vol. 40 (2005), p. 1032.

[16] A. Serrenho and A.F. Miguel: Journal of Porous Media Vol. 12 (2009), p. 1129

[17] FLUENT 6 User's Guide, Fluent Inc. (2003).

[18] T. Zhang and Q. Chen: Build. Environ. Vol. 42 (2007), p. 1675.

[19] Z. Zhang, X. Chen, S. Mazumdar, T. Zhang and Q. Chen: Build. Environ. Vol. 44 (2009), p. 85 . 